Yannik Schädler*, Michael Sorg, and Andreas Fischer

\title{
Data-based energy coverage measurements to discover the potentials of regional energy storage
}

\author{
Messdatenbasierte Bestimmung der Lastabdeckung zur Ermittlung des Potenzials regionaler \\ Energiespeicher
}

https://doi.org/10.1515/teme-2021-0115

Received November 21, 2021; accepted January 6, 2022

\begin{abstract}
The fluctuations of the renewable energies request storage systems as a significant part of the future energy system. Here we introduce a measurement approach regarding the regional coverage of the power demand and the influence of a storage system on it. The approach is based on measured power data with given uncertainties. The resolution is 15 minutes in the time domain and 2-digit ZIP code regions in the spatial domain. An idealized storage model is introduced, and one storage is implemented for each region. As a result, the regional coverage is measured for different storage parameters and the respective measurement uncertainty is assessed to be below the set goal of $2 \%$. Furthermore, the inverse problem, i. e. the storage dimensioning, is investigated by estimating the necessary storage parameters to achieve a desired regional coverage. Findings: electrical self-sufficiency can be reached in 23 out of 95 regions, in contrast in 56 regions the regional coverage is increased insignificantly regardless of the parameters of the storage system. This illustrates the necessity of power transport to enable the increase of regional coverage. Concluding, the proposed measurement approach enables the estimation of storage parameters and can be used for storage dimensioning.
\end{abstract}

Keywords: Storage dimensioning, renewable energy, measurement data, measurement approach.

Zusammenfassung: Die Schwankungen der erneuerbaren Energien erfordern Speichersysteme als wesentlichen Bestandteil des zukünftigen Energiesystems. Wir stellen daher ein Messprinzip zur Messung der regionalen Lastabdeckung des Strombedarfs und des Einflusses eines Speichersystems darauf vor. Das Messprinzip basiert auf gemessenen Leistungsdaten mit gegebenen Unsicherheiten.

\footnotetext{
*Corresponding author: Yannik Schädler, University of Bremen, Linzer Strasse 13, 28359 Bremen, Germany, e-mail: y.schaedler@bimaq.de, ORCID: https://orcid.org/0000-0003-2693-287X

Michael Sorg, Andreas Fischer, University of Bremen, Linzer Strasse 13, 28359 Bremen, Germany, ORCID:

https://orcid.org/0000-0001-7349-7722 (A. Fischer)
}

Die Leistungsdaten haben eine zeitliche Auflösung von 15 Minuten beziehen sich räumlich auf zweistellige Postleitzahlenregionen. Es wird ein idealisiertes Speichermodell eingeführt, und für jede Region wird ein Speicher implementiert. Als Ergebnis wird die regionale Lastabdeckung für verschiedene Speicherparameter gemessen, die Messunsicherheiten liegen dabei stets unter dem Ziel von $2 \%$. Darüber hinaus wird das inverse Problem, die Speicherdimensionierung, untersucht, indem die notwendigen Speicherparameter abgeschätzt werden, um eine gewünschte regionale Lastabdeckung zu erreichen. Ergebnisse: In 23 von 95 Regionen kann elektrische Autarkie erreicht werden, in 56 Regionen hingegen wird die regionale Lastabdeckung unabhängig von den Speicherparametern nur unwesentlich erhöht. Dies verdeutlicht die Notwendigkeit des Leistungstransports, um eine Erhöhung der Lastabdeckung zu ermöglichen. Zusammenfassend lässt sich sagen, dass das vorgeschlagene Messprinzip die Abschätzung von Speicherparametern ermöglicht und für die Speicherdimensionierung genutzt werden kann.

Schlagwörter: Speicherdimensionierung, regenerative Energie, Messdaten, Messprinzip.

\section{Introduction}

The $\mathrm{CO}_{2}$ concentration in the atmosphere continues to rise and increases the greenhouse effect. One of the biggest $\mathrm{CO}_{2}$ emission sources are power plants including coal and gas power plants [1] as they emitted $212 \mathrm{MtCO}_{2}$ of the total of $739 \mathrm{MtCO}_{2}$ emissions in Germany in 2020. The desired decarbonisation of the energy system requires an increasing renewable power feed-in such as from wind turbines and solar cells. However, the renewable power sources are fluctuating sources by nature. The fluctuations appear in the time domain, while the power density is inhomogeneuosly distributed in the spatial domain. In order to cope with the temporal power fluctuations while covering the power demand regionally, i. e. avoiding energy transports between different ZIP code regions, the difference between the fluctuating power production and power demand can 
be balanced locally with storage systems. For this purpose, a detailed study of the increase in regional demand coverage is needed. The measurement of the regionally covered power demand is thus required, which - in a second step - enables a study on the dimensioning of the power and capacity of storage systems that fulfills the following three requirements: First, the storage dimensioning is based on measured power time series with reasonable resolution. Second, the storage capacity as well as the maximum charging/discharging power of the storage system are included in the investigation. Third, the quality of the storage dimensioning results can be rated by an uncertainty assessment.

The dimensioning of storages has been investigated by [2] with a focus on combinations of storage systems and the estimation of their potential, but this investigation did not include any actual measured power time series. [3] did a numerical simulation for geotechnical storage planning with a focus on the northern German region, but did not include any measured power time series but estimated values. The investigation of [4] did focus on both parameters capacity and power of the storage, but only with power data from 2007 and data of two transformers in two distinct locations not representing an entire country. The work of [5] investigated the profitability of storage systems in combination with solar power, but used only weather data from one part of Germany and no power data such as power demand or wind power. Both investigations of [6] and [7] used real measured demand power time series, but focussed on the peak shaving capability of storage systems in an industrial environment and omitted calculating the uncertainties of the results. Lastly [8] investigated a combined usage of storage systems with power data from 100 dwellings with a focus on a higher economical revenue using control algorithms but not dimensioning the storage systems. As a result, a data-based capacity- and powerdimensioning of storage systems that includes actual time series and uncertainties of electric power is missing.

For this reason, the relation of the storage parameters capacity as well as maximum charging/discharging power with the regional coverage of the power demand is subsequently investigated on the basis of power measurement data. First, the regional coverage of the power demand is the output quantity of the considered measurement chain, while the capacity and the maximum charging/discharging power are the sole parameters of an idealized storage system. After this forward problem is solved for different storage parameters, the solution of the inverse problem is implicitely given. The research hypothesis reads: "The increase of regional coverage by storage systems with certain capacity and maximum charging/discharging power can be estimated with a storage model on the basis of the actual power data, i. e. with the measured values of power feed-in and power demand, with reasonable uncertainties below 2\%." Accordingly, the investigation is separated into two parts: The composition of a measurement chain for the regional coverage as a forward problem and the estimation of the storage parameters to reach a given regional coverage. Note that the quality of the investigation is assessed by deriving the uncertainty of the regional coverage at a given storage capacity and maximum charging/discharging power, and vice versa. Note further that the applied power database contains power values with a high spatiotemporal resolution, i. e. 15-minutes in the time domain and 2-digit ZIP code resolution in the spatial domain. Each of the 2-digit ZIP code regions has an average area of $3700 \mathrm{~km}^{2}$. The range of the database is Germany in the spatial domain and the years 2015 to 2019 in the time domain, respectively. The power values include solar power, wind power, power from biomass and water power plants, power demand and the conventional power sources hard and brown coal, nuclear power and power from oil and gas power plants.

The measurement principle for the regional coverage of the power demand as well as the principle for the estimation of the storage parameters is introduced in Section 2. In Section 3 follows a description of the implementation, including a presentation of the used power database. In Section 4 the results of the measurement of the regional coverage and the results of the estimation of the storage parameters are presented. Both results are also discussed in context of the uncertainty analysis that was conducted according to the "Guide to the expression of uncertainty in measurement" [9] in Section 5. The conclusions and the outlook are finally given in Section 6.

\section{Measurement principle}

\subsection{Measurement of the regional coverage}

The principle of measurement for the regional demand coverage is a data-based modelling of the charging and discharging process of storage systems. In particular, it is based on measured power data from the German energy system. The measurement chain for the measurand "regional demand coverage" is shown in Fig. 1 and begins with power sensors in the present energy system. The sensors measure the power feed-in of different energy sources and the power demand, respectively. Since not all of the 95 ZIP code regions are equipped with sensors for all types of 


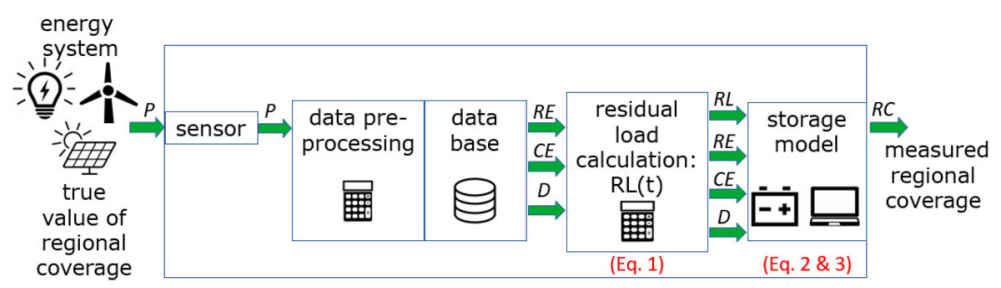

Figure 1: The measurement system consisting of sensors, data preprocessing, the database, the calculation of the residual loads RL(t) and the application of the storage model to determine the regional coverage $R C$. The arrows denote the flow of information, which are power data. The blocks denote either the measuring process in a sensor or the processing of the power data, e. g., in the storage model. The first arrow tagged with $P$ denotes the present power values in the energy system. The sensors measure the power values and deliver them to the data preprocessing, which is directly attached to the database. Next, the power values of demand $D$, renewable power feed-in $R E$ and conventional power feed-in $C E$ are used in the calculation of the residual loads following Eq. 1 . The residual loads and the power values are then used in the storage model to calculate the coverable power demand according to Eq. 2 and the regional coverage according to Eq. 3 The output of the measurement system is the measured value of the regional coverage introduced in Eq. 3 .

power time series, a data preprocessing fills regional gaps caused by missing or failing sensors with averaged values of their ZIP code neighbours. The data preprocessing also includes a scaling of the measured values in the regionally observed power plants up to the known installed power of all power plants in the considered region. The procedure is described in detail in [10]. After the data preprocessing, the data are stored in a database. Next, the residual load

$$
R L(r, t)=D(r, t)-R E(r, t)-C E(r, t),
$$

is calculated for each region $r$ and time $t$ as a measure of a power balance between the power production and consumption, which includes the measured conventional power data $C E$, the measured renewable power data $R E$ and the measured power demand $D$. The variable $r$ denotes the 2-digit ZIP code regions and ranges from 1 to 99 with four non-existent regions. Therefore, 95 different regions exist. The variable $t$ denotes the time interval in the considered year and ranges from 0 to 35040 , which is the number of 15-minute intervals in one year.

In order to describe the storage system in a mathematical fashion, it is distinguished between the total demand $D(r, t)$ and the actual regional coverable demand $\tilde{D}(r, t) . \tilde{D}(r, t)$ obeys the equation

$$
\tilde{D}(r, t)=\left\{\begin{array}{lll}
D(r, t) & \text { if } \quad & D(r, t) \leq P(r, t) \\
& & (\text { power surplus, charging) } \\
P_{\lim }(r, t) & \text { if } \quad & (r, t)>P(r, t) \\
& & \text { (power shortage, discharging) }
\end{array},\right.
$$

where $P(r, t)$ represents the sum of renewable and conventional power feed-in and $P_{\text {lim }}(r, t)$ represents the power limit that applies for the demand coverage and follows from the storages power limitations.

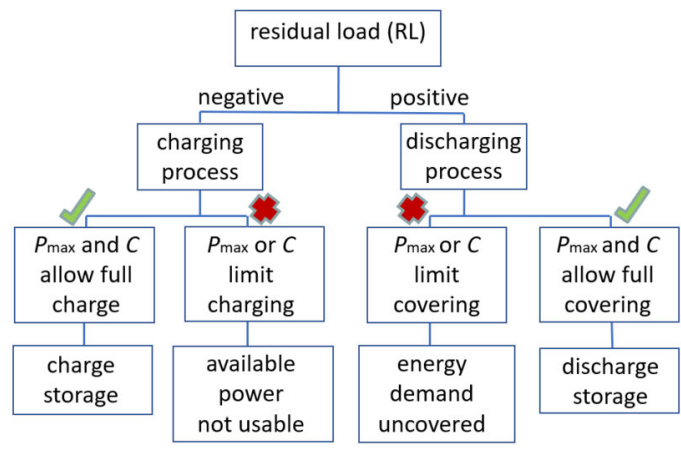

Figure 2: Flowchart of the storage model. The four conditions are checked for each 15-minute interval. If the capacity or the maximum power limits the charging or discharging process, either the available power is not usable or the demand can not be covered completely.

\subsection{Storage model}

The calculated residual loads in combination with the preprocessed power values are used further in the next step of the measurement chain, which is simulating the power storage with one storage unit per region. The storage system is modelled with the two essential parameters capacity $C$ and maximum charging/discharging power $P_{\max }$. The charge state of a storage system is the time- and regiondependent variable $C S(r, t)$. Both parameters are implemented as thresholds of the storage system that limit the charging and discharging process. The following conditions, that are also part of the decision tree that is visualized in Fig. 2, can limit the operation of the storage system (red crosses in Fig. 2): First, if the required power is higher than the maximum discharging power, $P_{\text {lim }}(r, t)$ from Eq. 2 is equal to the sum of the power inputs $P(r, t)$ and the maximum discharging power $P_{\max }$. Second, if the storage system is not adequately charged to satisfy 
the power shortage, $P_{\text {lim }}(r, t)$ is equal to the sum of the power input $P(r, t)$ and either the maximum discharging power $P_{\max }$ or the charge state $C S(r, t)$ that has to be converted to its respective power value by dividing the charge state by the length of the time interval. Whether the maximum discharging power $P_{\max }$ over the 15-minute interval or the charge state $C S(r, t)$ is added depends on the stored and, thus, available energy. The analogous conditions apply to the loading process. If the surplus power is greater than the maximum charging power $P_{\max }$, the surplus cannot be fully stored. Furthermore, the storage system must still have sufficient capacity to store the energy. However the loading process does not compromise the regional coverage as it only impairs the charge state $C S(r, t)$, but not the coverable demand $\tilde{D}(r, t)$. One particular challenge here becomes obvious: The resulting regional coverage depends on the history of the storage system.

The regional coverage $R C$ is defined and finally calculated as the sum of the regionally coverable power demand $\tilde{D}(r, t)$ over one year normalized by the total power demand $D(r, t)$ over one year in the considered region $r$ :

$$
R C(r)=\frac{\sum_{t} \tilde{D}(r, t)}{\sum_{t} D(r, t)} .
$$

This calculation is included in the storage block of the measurement chain, which provides the output of the measurement chain.

Note that the investigation is carried out with measurement data of the present energy system, which represent a large part, but not the whole energy system. However, this part is sufficient to investigate the effects in question here. Therefore, the totality of the measurement data can be called a "digital twin". Note further that a positive residual load represents a power shortage, where a negative residual load represents an power surplus. A shortage as well as a surplus is considered as an undesired event as a shortage leads to a power transport from other ZIP code regions or countries and a surplus leads to a curtailment of the power production, e.g. a curtailment of the wind harvest. The negative effects of power shortage and power surplus can be compensated with an appropriate storage system that has both the capacity $C$ and the maximum charging/discharging power $P_{\max }$ to either store or provide the respective energy. As the two storage parameters are highly important in the calculation of the regional coverage, their influence is of particular interest in the result section. Lastly, remember that the transport of energy between ZIP code regions was neglected in our study on purpose.

\subsection{Storage parameters}

In the second part of the investigation, the measurement chain from Fig. 1 is used inversely to estimate the necessary storage parameters in order to reach a desired regional coverage. The estimated capacity $\hat{C}$ and the estimated maximum charging/discharging power $\hat{P}_{\max }$ are denoted with a hat symbol. To examine the storage parameter dimensioning, we first define the following notation: The regional coverage without any storage system is written as $R C_{0}$ and the regional coverage reachable with a storage system with unlimited capacity and unlimited maximum charging/discharging power is written as $R C_{100}$ as a $100 \%$ reference of the possible benefit. Since a given goal can be achieved with different combinations of the two storage parameters, the notation is extended by the fixed parameter, that is either the capacity (e. g. $R C_{50, C=10 \mathrm{GWh}}$ ) or the maximum charging/discharging power (e. g. $R C_{50, P_{\max }=200 \mathrm{MW}}$ ). The estimated values for the parameters of interest are determined by choosing a very low and a very high value of the parameter as boundaries and then approaching the optimal parameter value that fulfills the desired regional coverage in an iterative and bisective fashion.

\section{Implementation of the measurement chain}

\subsection{Database}

The database consists of power values, measured by sensors in the present energy system. The sensors measure the power feed-in of renewable and conventional energy sources and the power demand, respectively. Each value represents the average power feed-in or power demand over a 15-minute period in one of the 95 ZIP code regions. In addition to the power demand, the available data includes the renewable power sources offshore wind power, solar power, biomass power and water power. Onshore wind power data is available only in 52 ZIP code regions, but has been completed to all 95 ZIP code regions using a model-based approach described in [10]. The conventional power sources also included in the database are hard and brown coal, nuclear power, oil and gas. The gap filling of regional and temporal power gaps ensures that values for all 95 ZIP code regions and for all 35040 intervals of the corresponding year are available. The regional gap filling is necessary for missing wind power values and uses 


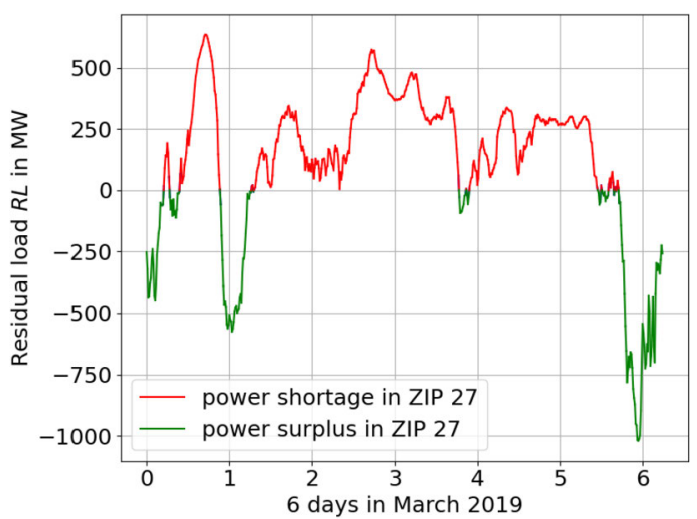

Figure 3: Example of a residual load time series in ZIP code region 27. Positive and negative values appear as denoted in red and green. Positive values represent power shortage, negative values represent power surplus. With adequate storage capacity and maximum power, the power surplus can be used to balance a part of the power shortage.

three steps to estimate the wind power feed-in for the region of interest. At first all present values are normalized to the observed wind power capacity in the respective region. Secondly the average of normalized values of all ZIP code neighbours is used to estimate the normalized wind power feed-in for the region of interest. Thirdly the normalized value is scaled up to the real wind power feed-in with the installed wind power capacity. A temporal gap filling is applied for missing solar power values, which uses linear splines between present values as only small gaps for individual ZIP code regions appear. The power measurement database is a continuation of the database introduced in the publications of [10] and [11]. It consists of power measurement data of the years 2015 to 2020, but the latest year with full datasets on all renewable powers, conventional powers and power demand is currently 2019 . Therefore the investigation here is focused on the power time series from the year 2019. As an example, a few days of the time series of the residual load of ZIP code region 27, which is the surrounding countryside of the north-west German city Bremen, is shown in Fig. 3. In the exemplary diagram the necessity for storage systems are presented as the residual load $R L$ has positive as well as negative values. In the example it becomes visible that the residual load has positive as well as negative values over the course of six days. This reinforces the suggestion that storage systems should be used to balance the residual load.

\subsection{Implementation of the storage model}

The technical characteristics of storage systems can generally be described by the parameters capacity, maximum charging and discharging power, losses in the charging and discharging process, temperature-dependent parameters and deterioration parameters. The idealized storage model used here is limited to the parameters capacity $C$, and maximum charging and maximum discharging powers that are assumed to be equal and therefore named maximum charging/discharging power $P_{\max }$. Both parameters are implemented as thresholds of the storage system. Storage losses are not considered. The storage system can only be charged with a power surplus $P$ if both conditions are fulfilled:

- The capacity allows the charge with the corresponding amount of energy $t_{\text {interval }} \cdot P$ with $t_{\text {interval }}=15 \mathrm{~min}$.

- The maximum charging/discharging power $P_{\max }$ of the storage system allows the charge or discharge with the power $P$ in the respective interval.

For the discharging of storage systems the analogous conditions apply: The energy discharged is not limited to the capacity of the storage system, but by its charging state $C S$.

\subsection{Determination of the storage parameters}

In order to estimate one of the parameters $\hat{C}$ and $\hat{P}_{\text {max }}$ necessary to reach a goal value of the regional coverage $R C$, the other parameter is set to a fixed value first, e. g. if we are interested in the necessary capacity to reach $\mathrm{x} \%$ of the maximum possible benefit of regional coverage $R C_{\chi}$, the value of $P_{\max }$ is set to a given value first. Next, the goal value of the benefit in regional coverage is set, e.g. $10 \%$ of the maximum possible benefit, in the defined notation $R C_{10}$. To determine the estimated value $\hat{C}$, first one value that is much too small $C_{\text {small }}$ and one that is much too large $C_{\text {large }}$ is considered. The two values for the regional coverage are obtained and the one of them is with a smaller distance to the goal is identified. In the next iteration, the boundary value with a smaller distance to the goal is used together with the mean of the two boundaries as a new set of boundaries. The algorithm stops after two consecutive capacity values have a relative difference below $0.01 \%$. For the estimation of the maximum charging/discharging power $\hat{P}_{\max }$ the same bisective algorithm is applied.

\section{Results}

If not indicated, ZIP code region 27 is subsequently considered for an exemplary presentation and discussion of the results. As a first result, the residual load with and 


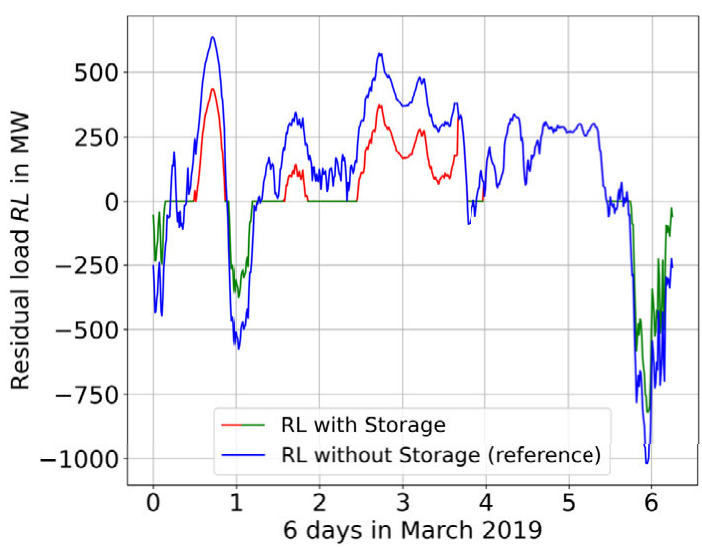

(a)

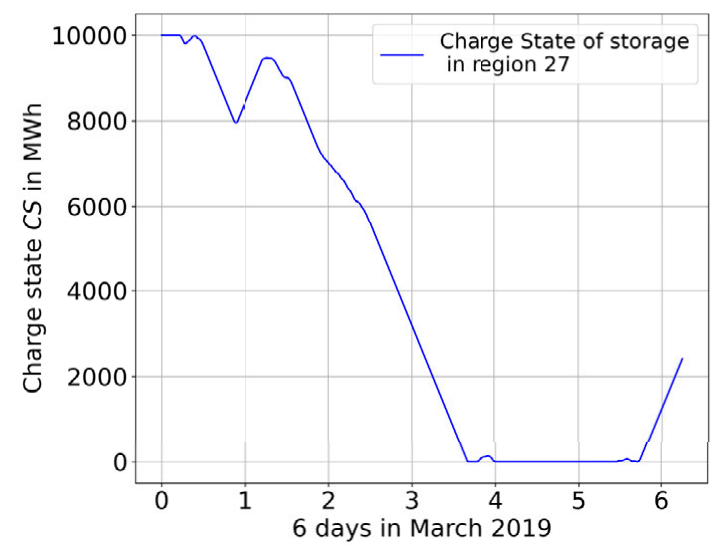

(b)

Figure 4: (a) Original residual load of ZIP code region 27 and residual load with a storage system with 10 GWh capacity and 200 MW maximum charging/discharging power. Green indicates intervals without a power shortage, red indicates a power shortage that needs to be covered with additional controllable power plants. The storage cannot balance the negative residual loads as the maximum charging power is not high enough. The positive ones are not balanced completely as the discharging power is not high enough. (b) The charge state of the corresponding storage system. In the beginning of the considered time period the storage is fully charged but gets discharged completely over the course of roughly three days.

without a storage system is calculated and plotted together with the respective charge state in Fig. 4 over the same six days as in Fig. 3. The storage system used here has the parameters $C=10 \mathrm{GWh}$ and $P_{\max }=200 \mathrm{MW}$. As expected, the residual load shows decreased positive values and increased negative values due to the charging/discharging process of the storage.

Next, the regional coverage is calculated for ZIP code region 27 with a storage system of $10 \mathrm{GWh}$ capacity and 200 MW maximum charging/discharging power. In this case, the regional coverage is $(85.7 \pm 1.2) \%$ compared to $(77.4 \pm 1.1) \%$ without storage. Since a storage system with unlimited capacity and charging/discharging power enables a complete regional demand coverage, the regional coverage of the storage system evaluated here corresponds to $R C_{25}$, i. e. $25 \%$ of the maximum reachable regional coverage with unlimited storages.

In a third step, the capacity of the storage system is varied to investigate its influence on the regional coverage. This results in a sigmoidal curve in a semi-logarithmic diagram as shown in Fig. 5. Here, depending on the storage capacity, is clearly visible as the regional coverage $R C$ can be increased from $77 \%$ to $100 \%$.

In a fourth investigation the maximum charging/discharging power $P_{\max }$ is varied also, and the resulting regional coverage curves are plotted over the capacity in Fig.6. As a result, both the capacity and the maximum charging/discharging power must be of sufficient size to access the full storage potential for increasing the regional coverage. The results also suggest that the capacity with

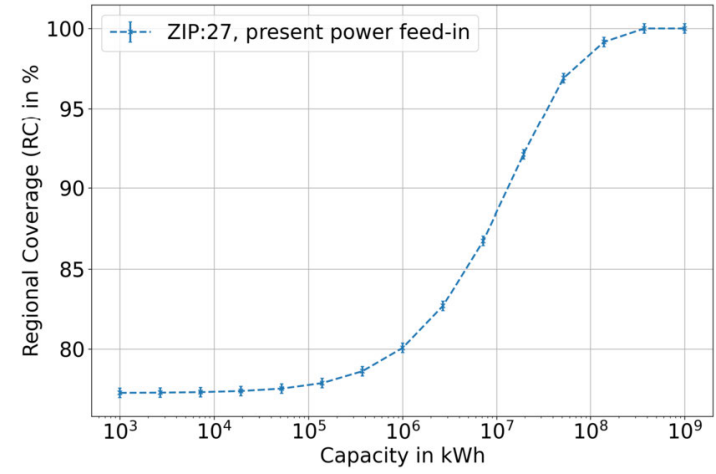

Figure 5: Results for ZIP region 27 and for different storage capacities. The maximum charging/discharging power was unlimited in this example. Note that $\approx 77 \%$ of power demand is covered even without any storage systems and $100 \%$ of regional coverage can be achieved with an adequate storage.

the highest slope is in the same order of magnitude as the typical daily power demand.

Lastly, the inverse problem of identifying the necessary capacity of a storage system to reach a benefit in regional coverage of $50 \%$ of the maximum possible coverage increase is considered. As given maximum charging/discharging power $P_{\max }=200 \mathrm{MW}$ is set. With the notation defined in Section 3.3 the corresponding residual coverage goal can be written as $R C_{50, P_{\max }=200 \mathrm{Mw}}$. The iterative process starts with one value that is much too small $C_{\text {small }}=0 \mathrm{kWh}$ and one that is much too large $C_{\text {large }}=$ $10^{10} \mathrm{kWh}$. After a few iterations, the resulting estimate is $\hat{C}=51.40 \mathrm{GWh}$. 


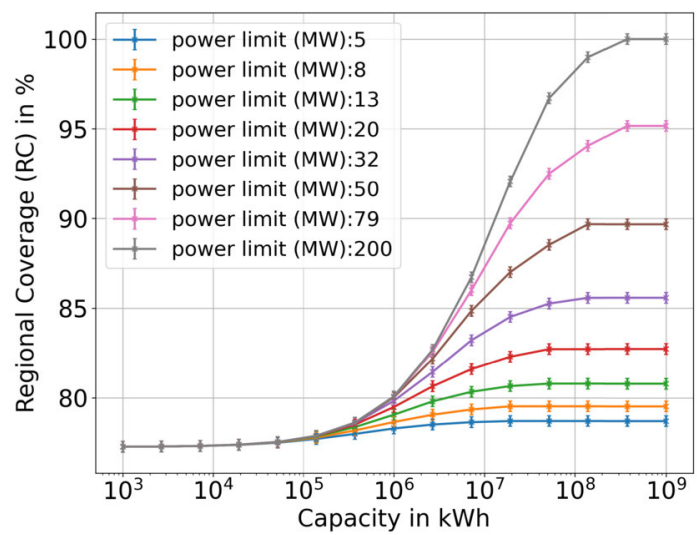

Figure 6: Example for different maximum charging/discharging power values. Different maximum regional coverage is clearly visible and ranges from $78 \%$ to $100 \%$. Interestingly, all curves have in common that the most prominent increase takes place between a capacity of $50 \mathrm{GWh}$ and $500 \mathrm{GWh}$, which corresponds roughly to the average daily energy demand. This provides an indication that balancing the daily fluctuations is most beneficial regarding the regional coverage.

\section{Quality discussion}

The quality of the results can be assessed by analogy with the "Guide to the expression of uncertainty in measurement"(GUM) [9]. For this purpose, a Monte Carlo simulation is performed while each power value $P^{\sim}$ in the database is assumed to lie between the upper boundary of $P^{\sim+}=1.05 \cdot P^{\sim}$ and the lower boundary of $P^{\sim-}=0.95 \cdot P^{\sim}$. The tilde represents all types of power input and power demand. Since there is no further knowledge on the probability distribution of the values, the probability distribution is chosen to be a uniform one following the principle of maximum entropy suggested by the GUM. The associated standard uncertainty of the values $P^{\sim}$ obeys the equation

$$
u\left(P^{\sim}\right)=\sqrt{\frac{\left(0.1 \cdot P^{\sim}\right)^{2}}{12}} \approx 0.029 \cdot P^{\sim} .
$$

The uncertainty for each calculated value of the regional coverage $R C$ is assessed by repeating the measurement 100 times with perturbed power values. The resulting uncertainties ranging from $0.01 \%$ to $0.5 \%$ are plotted in the diagrams in respective results in Figs. 5 and 6 as errorbars. As the relative uncertainties are smaller than the required 2\% defined in the research hypothesis, the databased measurement of the regional coverage of power demand is achieved successfully.

Lastly, the uncertainty of the regional coverage is used to estimate, as an example, the uncertainty of the estimated parameter $\hat{C}$ that is necessary to reach
$R C_{50, P_{\max }=200 \mathrm{MW}}$. This is accomplished by calculating the slope $m$ of the local characteristic curve with

$$
m=\frac{\partial R C}{\partial \hat{C}},
$$

and using it to propagate the uncertainty of the regional coverage on the storage parameter. The final equation for the combined standard uncertainty is

$$
u_{c}(\hat{C})=m^{-1} \cdot u(R C)
$$

The uncertainty of the estimation derived here yields an uncertainty of $u(\hat{C})=0.087 \mathrm{GWh}$. Finally, the complete result of the estimation reads $\hat{C}=(51.40 \pm 0.09) \mathrm{GWh}$. The analogous approach can be used for the calculation of the uncertainty of $\hat{P}_{\text {max }}$. The determination of the uncertainties of the storage parameters enable a consideration of the financial uncertainties of the expansion of storage systems.

\section{Conclusion and outlook}

A data-based modelling of the charging and discharging of storage systems and its influence on the regional coverable power demand is conducted. The modelling is based on measured data from the present German energy system. In a second step, the measurement of the regional coverage is used to estimate the necessary storage parameters to result in a desired regional coverage of power demand. After the method, the database and the idealized storage model are introduced, the results are presented. Firstly, the regional coverage was measured with a storage system with set parameters. Secondly, the parameters were varied to investigate their influence on the regional coverage. In all resulting values for the regional coverage, the uncertainty was below the defined goal of $2 \%$ for an assumed maximum relative uncertainty of $\pm 5 \%$ of the power value entries in the data base, and thus the residual load $R L$. Lastly, the inverse problem was investigated: A desired regional coverage was given and the task of estimating one of the two storage parameters was resolved, even though the relative uncertainty does not correspond to the specified target. The presented method of modelling the storage processes data driven and evaluating the regional coverage is thus a helpful measurement tool for the transformation of the German energy system.

In future investigations, the approach can support the dimensioning of storage systems regarding their power and capacity. In order to model the storages more realistically, more parameters must be taken into account, e.g. 
aging processes and the efficiency of the charging and discharging processes. In order to model and optimize the full energy system and expansions made necessary by the growing renewable power feed-in, the other mechanisms such as power transport must be modelled in addition to the storage of power. At last, the uncertainties described here have a financial equivalent that must be investigated in order to estimate if the further expansion of storage systems is economically reasonable. One promising approach for the expansion and operation of the future energy system is then the usage of machine-learning methods, in particular neural networks, as the process correlations in the distribution network can likely be answered with a trained neural network. The neural networks can also be trained with differently scaled scenarios from the database of measured data.

Funding: The authors gratefully acknowledge the Federal Ministry for Economic Affairs and Energy (BMWi) for funding the project "Analysis for the geographic distribution of wind and solar generation and their influences on the power grid (GEOWISOL2)”, reference number 0350033A.

\section{References}

1. German Federal Environmental Agency. Emission sources, 2020. URL https://www.umweltbundesamt.de/themen/klimaenergie/treibhausgas-emissionen/emissionsquellen.

2. Sebastian Günther, Astrid Bensmann, and Richard Hanke-Rauschenbach. Theoretical dimensioning and sizing limits of hybrid energy storage systems. Applied Energy, 210: 127-137, 2018. ISSN 0306-2619. https://doi.org/10.1016/j.apenergy.2017.10.116.

3. Alina Kabuth, Andreas Dahmke, Christof Beyer, Lars Bilke, Frank Dethlefsen, Peter Dietrich, Rainer Duttmann, Markus Ebert, Volker Feeser, Uwe-Jens Görke, et al. Energy storage in the geological subsurface: dimensioning, risk analysis and spatial planning: the angus+ project. Environmental Earth Sciences, 76 (1): 1-17, 2017.

4. Nicholas Etherden and Math H. J. Bollen. Dimensioning of energy storage for increased integration of wind power. IEEE Transactions on Sustainable Energy, 4 (3): 546-553, 2013. 10.1109/TSTE.2012.2228244.

5. Andreas Zucker and Timothée Hinchliffe. Optimum sizing of pv-attached electricity storage according to power market signals - a case study for germany and italy. Applied Energy, 127: 141-155, 2014. ISSN 0306-2619. https://doi.org/10.1016/j.apenergy.2014.04.038.

6. Christopher Lange, Alexandra Rueß, Andreas Nuß, Richard Öchsner, and Martin März. Dimensioning battery energy storage systems for peak shaving based on a real-time control algorithm. Applied Energy, 280: 115993, 2020. ISSN 0306-2619. https://doi.org/10.1016/j.apenergy.2020.115993.

7. Rodrigo Martins, Holger C. Hesse, Johanna Jungbauer, Thomas Vorbuchner, and Petr Musilek. Optimal component sizing for peak shaving in battery energy storage system for industrial applications. Energies, 11 (8), 2018. ISSN 1996-1073. 10.3390/en11082048.

8. David Parra and Martin K. Patel. The nature of combining energy storage applications for residential battery technology. Applied Energy, 239: 1343-1355, 2019. ISSN 0306-2619. https://doi.org/10.1016/j.apenergy.2019.01.218.

9. JCGM. Jcgm 100: Evaluation of measurement data - guide to the expression of uncertainty in measurement. Technical report, JCGM, 2008.

10. Yannik Schädler, Volker Renken, Michael Sorg, Lewin Gerdes, Gerhard Gerdes, and Andreas Fischer. Power transport needs for the german power grid for a major demand coverage by wind and solar power. Energy Strategy Reviews, 34: 100626, 2021.

11. Volker Renken, Michael Sorg, Volker Marschner, Lewin Gerdes, Gerhard Gerdes, and Andreas Fischer. Geographical comparison between wind power, solar power and demand for the german regions and data filling concepts. Renewable Energy, 126: 475-484, 2018.

\section{Bionotes}

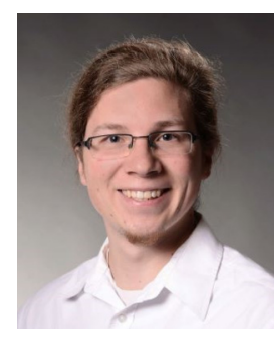

Yannik Schädler

University of Bremen, Linzer Strasse 13, 28359 Bremen, Germany

y.schaedler@bimaq.de

Yannik Schädler studied physics at the University of Bremen with a focus on theoretical physics before working 6 months in a software company and finally moving to the Bremen Institute for Measurement, Automation and Quality Science (BIMAQ). He is the project coordinator of the research project GEOWISOL2, which aims to build a database of power input and demand in a high resolution to use in data based modelling approaches for energy transport and storage.

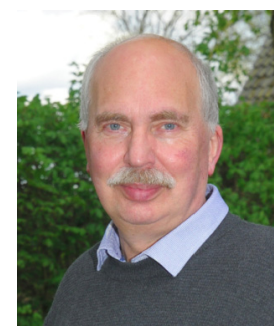

Michael Sorg

University of Bremen, Linzer Strasse 13, 28359 Bremen, Germany m.sorg@bimaq.de

Michael Sorg studied electrical engineering at the University of Karlsruhe and at the University of Bremen with a focus on communications engineering. After his research period at the Institute for Measurement, Control and Systems Engineering at the University of Bremen, he moved in 1998 to the Bremen Institute for Measurement, Automation and Quality Science (BIMAQ) where he heads the research group Energy Systems and Materials Testing. 


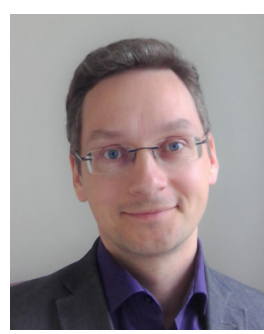

\section{Andreas Fischer}

University of Bremen, Linzer Strasse 13, 28359 Bremen, Germany

andreas.fischer@bimaq.de

Andreas Fischer received his diploma in electrical engineering in 2004 and completed his PhD at Dresden University of Technology in 2009. While working as head of the "Measurement Systems Engineering" group in the Department of Measurement and Sensor Systems Engineering (2009-2016), he completed his habilitation in 2014. Since 2016, he has been a professor in the Department of Production Engineering at the University of Bremen and head of the Bremen Institute for Measurement, Automation and Quality Science (BIMAQ). His research areas include measurement systems engineering, optical measurement principles for flow and production metrology as well as the application of model-based measurement systems and the investigation of their measurability limits. 